Document downloaded from:

http://hdl.handle.net/10251/155059

This paper must be cited as:

Tello-Oquendo, L.; Pla, V.; Leyva-Mayorga, I.; Martínez Bauset, J.; Casares-Giner, V.; Guijarro, L. (2019). Efficient Random Access Channel Evaluation and Load Estimation in LTE-A with Massive MTC. IEEE Transactions on Vehicular Technology. 68(2):1998-2002. https://doi.org/10.1109/TVT.2018.2885333

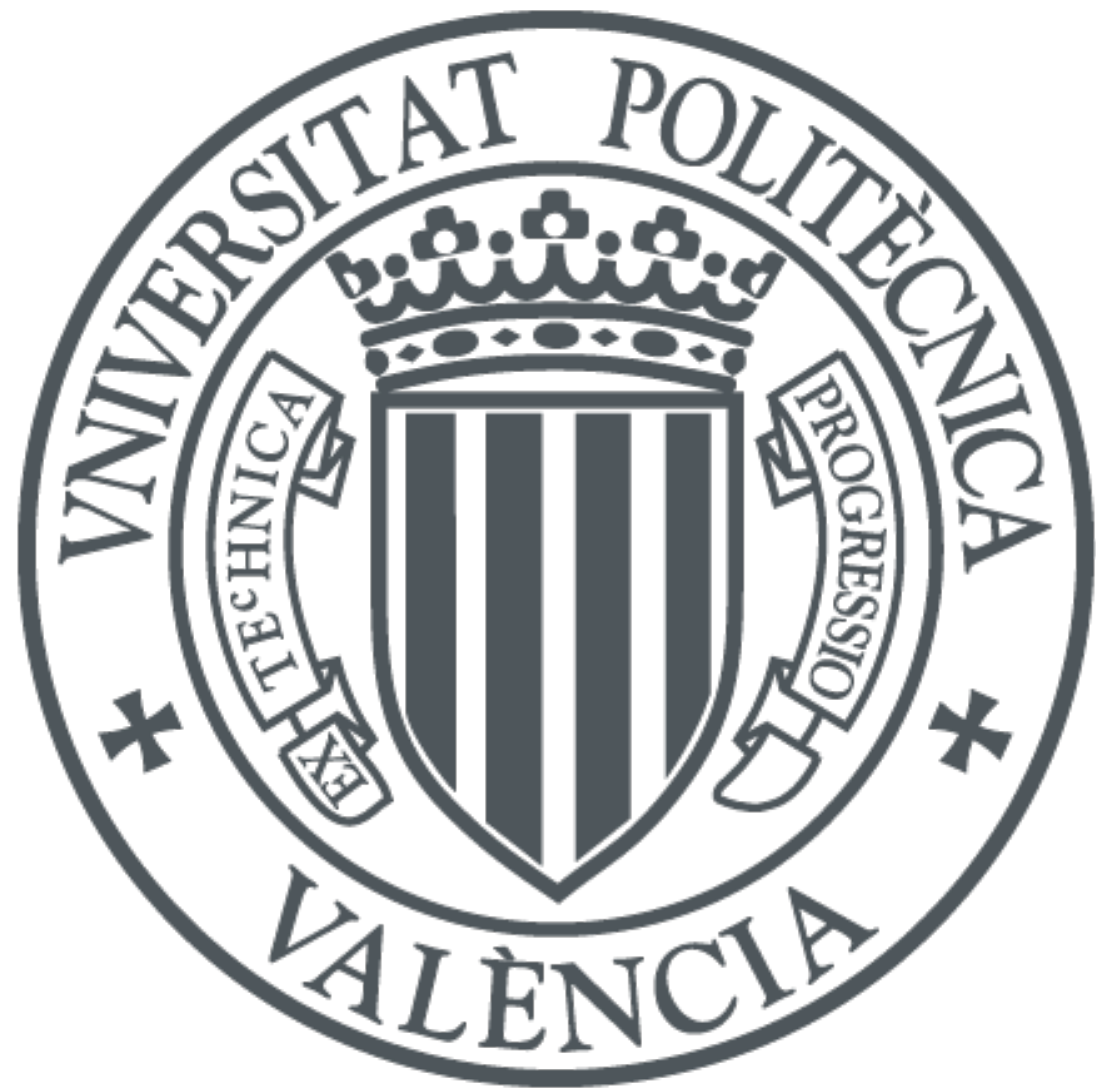

The final publication is available at

https://doi.org/10.1109/TVT.2018.2885333

Copyright Institute of Electrical and Electronics Engineers

Additional Information

(C) 2019 IEEE. Personal use of this material is permitted. Permissíon from IEEE must be obtained for all other uses, in any current or future media, including reprinting/republishing this material for advertisíng or promotional purposes, creating new collective works, for resale or redistribution to servers or lists, or reuse of any copyrighted component of this work in other works." 


\title{
Efficient Random Access Channel Evaluation and Load Estimation in LTE-A With Massive MTC
}

\author{
Luis Tello-Oquendo, Member, IEEE, Vicent Pla, Israel Leyva-Mayorga, Jorge Martinez-Bauset, \\ Vicente Casares-Giner, Life Member, IEEE, and Luis Guijarro
}

\begin{abstract}
The deployment of machine-type communications (MTC) together with cellular networks have great potential to create the ubiquitous Internet of Things environment. Nevertheless, the simultaneous activation of a large number of MTC devices (UEs) is a situation difficult to manage at the evolved Node B (eNB). The knowledge of the joint probability distribution function (PDF) of the number of successful and collided access requests within a random access opportunity (RAO) is a crucial piece of information for contriving congestion control schemes. A closed-form expression and an efficient recursion to obtain this joint $\mathrm{PDF}$ are derived in this paper. Furthermore, we exploit this PDF to design estimators of the number of contending UEs in a RAO. Our numerical results validate the effectiveness of our formulation and show that its computational cost is considerably lower than that of other related approaches. In addition, our estimators can be used by the eNBs to implement highly efficient congestion control methods.
\end{abstract}

Index Terms-Cellular systems, machine-type communications (MTC), random access channel (RACH).

\section{INTRODUCTION}

Nowadays, the use of cellular network technologies such as long term evolution advanced (LTE-A) and beyond for providing machine-type communications (MTC) has attracted significant attention from both the research community and the industry. The widely deployed infrastructure is a major driving force that motivates MTC application developers to adopt cellular networks for their numerous remote monitoring and controlling applications [1], [2]. However, critical problems like congestion and overload of radio access and core networks need to be addressed for efficient cellular MTC [3], [4].

In LTE-A, when an MTC device (named UE herein) wants to access the cellular network, it performs a random access procedure. The random access channel $(\mathrm{RACH})$ is used to signal the connection request; it is allowed in predefined time/frequency resources, hereafter random access

Manuscript received xxxx xx, 2017; revised xxxx xx, 2018. This work was supported in part by the Ministry of Economy and Competitiveness of Spain under Grant TIN2013-47272-C2-1-R and Grant TEC2015-71932REDT. The work of L. Tello-Oquendo was supported in part by the Universitat Politècnica de València under the Programa de Ayudas de Investigación y Desarrollo (PAID). The work of I. Leyva-Mayorga was supported in part by the CONACYT-Gobierno del Estado de México under Grant 383936.

Copyright (c) 2015 IEEE. Personal use of this material is permitted. However, permission to use this material for any other purposes must be obtained from the IEEE by sending a request to pubs-permissions@ieee.org.

The authors are with the ITACA Institute, Universitat Politècnica de València, Valencia 46022, Spain. L. Tello-Oquendo is now with the College of Engineering, Universidad Nacional de Chimborazo, Riobamba 060108, Ecuador. (e-mail: luis.tello@unach.edu.ec, \{vpla, isleyma, jmartinez, vcasares\}@upv.es).

Digital Object Identifier 10.1109/TVT.2018.... opportunities (RAOs) [5], [6]. The evolved Node B (eNB) has a number of preambles available for initial access to the network. These preambles are generated by Zadoff-Chu sequences due to their good correlation properties [6], [7] and are transmitted by the UEs for attempting the first access to the network.

A four-message handshake is performed in the contentionbased random access. In Msgl, a UE transmits a randomly chosen preamble from the preamble pool during one of the available RAOs. A preamble will be detected at the eNB if it has not been chosen by more than one UE in the same RAO. Otherwise, a collision occurs. Then, the eNB sends a random access response message, $M s g 2$, which includes one uplink grant for each detected preamble. $M s g 2$ is used to assign timefrequency resources to the UEs for the transmission of Msg3. UEs wait for a predefined time window to receive the uplink grant. If no uplink grant is received by the end of this window and the maximum number of access attempts has not been reached, the UEs wait for a random time and then perform a new access attempt. That is, they select a new preamble and transmit it at the next RAO. The UEs that receive an uplink grant send their connection request message, $M s g 3$, using the resources specified by the eNB. Finally, the eNB responds to each Msg3 transmission with a contention resolution message, Msg4. The interested reader is referred to [5], [8]-[11] for further details.

The main contributions of this study are the following:

- We propose a closed-form expression for the joint probability distribution function (PDF) of the number of successful and collided preamble transmissions within a RAO.

- We devise a computationally efficient recursion to compute the mentioned joint PDF even when the number of contending UEs is large.

- We compare the computational cost of both, our closedform expression and recursive approach with that of other proposed methods, which only obtain the marginal distribution of successful attempts, and show that not only our approach yields a result that provides more information (joint vs. marginal PDF), but our recursion is computationally far less expensive.

- We use the joint PDF to design a series of estimators of the number of contending UEs in a RAO based on the maximum likelihood and Bayesian approaches.

The expressions we obtain are useful also in other contexts, where multiple resources (such as slots, channels) are randomly accessed by a pool of users as noted by the 
authors in [12]. In fact, the contention-based random access procedure detailed above is similar to the slotted ALOHA protocol [13]. After the preamble transmission in Msgl, the eNB can distinguish a request only if a preamble was transmitted by a single device (i.e., a UE can be connected if there is no access collision). In this sense, the random access procedure can be seen as a multi-channel ALOHA [12], [14], [15], where congestion control is tackled by estimating the number of arrivals or the number of UEs that send preambles to the eNB [16].

The remainder of the paper is organized as follows. Section II presents the most relevant related work in this subject. Section III introduces a closed-form expression and an efficient recursion to find the joint PDF of the number of successful and collided preambles in a RAO. Additionally, we use this PDF to design a series of estimators of the number of contending UEs. Section IV shows the numerical results and showcases the efficacy of our contributions. Finally, Section V draws the conclusions.

\section{RELATED WORK}

Random access is identified as a key issue in MTC. In recent studies, approaches using non-trivial combinatorics have been developed to derive the PDF of the number of successful UEs in one-shot random access in multi-channel ALOHA.

In [12], [17], Wei et al. derived an explicit expression for computing the PDF of the number of successful transmissions. It is based on balls-and-bins combinatorics and uses an extension of the Stirling numbers of the second kind. In [18], Duan et al. provide another approach to compute the PDF of the number of successful preamble transmissions using combinatorial analysis. In [19], Arouk et al. provide another expression to compute the PDF of the number of successful preamble transmissions using the balls-and-bins approach and mathematical induction. These publications evince a current interest in deriving closed-form mathematical expressions to study the RACH in modern cellular networks using analytical models. However, previous proposals result in complex formulations and, as shown later, some of them fail to provide consistent results when the number of UEs increases beyond a certain value, severely limiting their practical applicability.

In addition, the PDF of the number of collided preamble transmissions is not addressed in previous studies. However, as shown later, the joint PDF of the number of successful and collided attempts plays a major role in the design of accurate estimators of the number of contending UEs in a RAO.

In this paper, to fully address the above mentioned issues, we devise: 1) a simpler closed-form expression for computing the joint PDF of the number of successful and collided transmission attempts; and 2) an efficient recursion for the same purpose that substantially reduces the computational cost.

\section{JOINT PDF OF THE NUMBER OF SUCCESSFUL AND Collided Preamble Transmissions}

This section is organized in three parts. Section III-A presents the closed-form expression of the PDF, Section III-B presents a computationally efficient recursion to compute the
PDF, and Section III-C presents an application of the PDF to design a series of estimators of the number of contending UEs.

For the formulation, we focus on a single RAO. Let $r$ be number of available preambles. Also, let $n$ be the number of contending UEs in a RAO (i.e., the UEs that transmit a preamble selected among the $r$ available preambles with equal probability). Finally, let $s$ be the number of preambles selected by exactly one UE, and $c$ the number of collided preambles. We consider pairs $(s, c)$ in the set $\mathcal{R}_{n} \triangleq\left\{(s, c) \in \mathbb{N}^{2} \mid s+c \leq\right.$ $r, s+\beta c=n, \beta \geq 2\}$.

\section{A. Closed-Form Expression}

Lemma 1: Consider $n$ UEs that independently choose a single preamble from a set of $c$ different preambles with equal probability. Let $\mathrm{F}(n, c)$ denote the probability that at least two UEs are assigned to each preamble. Then, $\mathrm{F}(n, c)=0$ if $n<2 c$. For $n \geq 2 c$ we have

$$
\mathrm{F}(n, c)=1+\sum_{k=1}^{c-1}(-1)^{k}\left(\begin{array}{l}
c \\
k
\end{array}\right) \sum_{m=0}^{k} p_{m, k},
$$

where

$$
\begin{aligned}
p_{m, k} & \triangleq\left(\begin{array}{c}
n \\
m
\end{array}\right)\left(\frac{k}{c}\right)^{m}\left(1-\frac{k}{c}\right)^{n-m} \frac{k}{k} \frac{k-1}{k} \cdots \frac{k-(m-1)}{k} \\
& =\left(\begin{array}{c}
n \\
m
\end{array}\right)\left(\frac{k}{c}\right)^{m}\left(1-\frac{k}{c}\right)^{n-m} \frac{k !}{(k-m) ! k^{m}}
\end{aligned}
$$

Proof: In what follows, we focus on the case $n \geq 2 c$. Consider a subset of $k<c$ preambles. The probability that exactly $m$ UEs $(m=1, \ldots, k)$ are assigned to the preambles in this subset, with at most one UE per preamble, is given by (2). Moreover, it is easy to check that the right-hand side of (2) is also valid for $m=0$.

Let $A_{k}$ denote the event that less than two (i.e., one or none) UEs have been assigned to the $k$ th preamble. Then, we can write

$$
\begin{aligned}
\mathrm{F}(n, c) & =1-\operatorname{Pr}\left(A_{1} \cup A_{2} \cup \cdots \cup A_{c}\right) \\
& =1-\sum_{k=1}^{c}(-1)^{k-1} \sum_{1 \leq i_{1}<\cdots<i_{k} \leq c} \operatorname{Pr}\left(\bigcap_{j=1}^{k} A_{i_{j}}\right) \\
& =1-\sum_{k=1}^{c-1}(-1)^{k-1} \sum_{1 \leq i_{1}<\cdots<i_{k} \leq c} \sum_{m=0}^{k} p_{m, k} \\
& =1+\sum_{k=1}^{c-1}(-1)^{k}\left(\begin{array}{l}
c \\
k
\end{array}\right) \sum_{m=0}^{k} p_{m, k} .
\end{aligned}
$$

Above we have used the fact that $\operatorname{Pr}\left(\bigcap_{j=1}^{c} A_{i_{j}}\right)=0$, since $n \geq 2 c$.

Theorem 1: The conditional joint probability of having exactly $s$ successes and $c$ collisions, when $n$ UEs transmitted 
their preambles, is given by

$$
\begin{aligned}
& P_{n}(s, c)=
\end{aligned}
$$

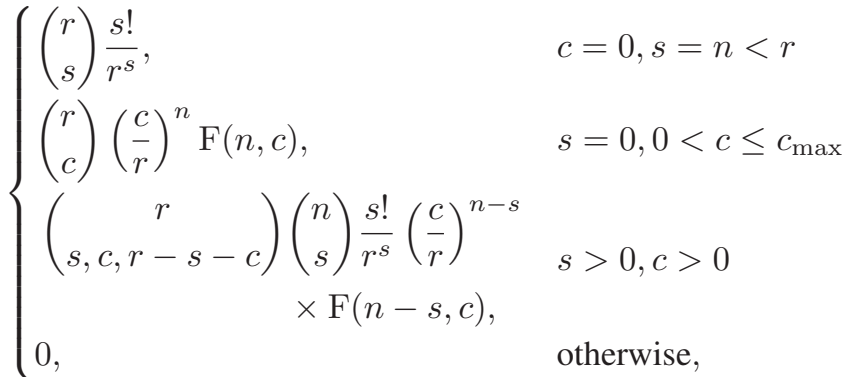

where $c_{\max }=\min \{r,\lfloor n / 2\rfloor\}$.

Proof:

1) $c=0, s=n<r$

Given a fixed set of $s$ preambles, the probability that $n=s$ UEs choose these $s$ preambles without collision is given by

$$
\frac{s}{r} \times \frac{s-1}{r} \times \cdots \times \frac{2}{r} \times \frac{1}{r}=\frac{s !}{r^{s}} .
$$

Taking into account that the number of different subsets of $s$ preambles is $\left(\begin{array}{l}r \\ s\end{array}\right)$, we have the first case in (4).

2) $s=0, c \leq c_{\max }$

Given a fixed set of $c$ preambles, the probability that $n$ UEs choose a preamble within this set is $(c / r)^{n}$. Using $\mathrm{F}(n, c)$ [see (3)] to account for the probability that each of the $c$ preambles is selected by at least two of the $n$ UEs, and taking into account the number of different subsets of $c$ preambles, we have the second case in (4).

3) $s>0, c>0$

Consider a selection of preambles performed by $n$ UEs resulting in $s$ successes (selected by exactly one UE), $c$ collisions (selected by at least two UEs), and $r-n-c$ unused preambles.

The probability that $s$ out of the $n$ UEs choose a preamble without collision is $\left(\begin{array}{l}n \\ s\end{array}\right) \frac{s !}{r^{s}}$.

The probability that $n-s$ out of the $n$ UEs choose a preamble with collision is $\left(\frac{c}{r}\right)^{n-s} \times \mathrm{F}(n-s, c)$.

Taking into account that the number of different preamble selections is $\left(\begin{array}{c}r \\ s, c, r-s-c\end{array}\right)$, we have the third case in (4).

Finally, the marginal probability distributions can be computed as

$$
\begin{aligned}
& P_{n}(s) \triangleq \operatorname{Pr}(s \mid n)=\sum_{c=0}^{c_{\max }} P_{n}(s, c) \\
& P_{n}(c) \triangleq \operatorname{Pr}(c \mid n)=\sum_{s=0}^{\min \{r, n\}} P_{n}(s, c) .
\end{aligned}
$$

\section{B. Recursion}

Although (4) is a simple expression, the computation of factorials might require a high computational cost when a massive number of UEs access the network. This limitation is also present in [12], [18], [19]. To overcome this limitation, we devise the following recursion

$$
\begin{aligned}
P_{n}(s, c)= & \frac{r-(s-1+c)}{r} P_{n-1}(s-1, c) \\
& +\frac{s+1}{r} P_{n-1}(s+1, c-1)+\frac{c}{r} P_{n-1}(s, c),
\end{aligned}
$$

where $P_{0}(0,0)=1$, and $P_{n}(s, c)=0$ if $(s, c) \notin \mathcal{R}_{n}$.

Clearly, from the distribution for $n-1$ UEs, we obtain the distribution when a UE is added. Let $(s, c)_{n}$ represent the case in which $n$ UEs have chosen their preambles leading to $s$ successes and $c$ collisions. The three outcomes that can lead to $(s, c)_{n}$ are:

1) Being the system in $(s-1, c)_{n-1}$, the $n$th UE chooses one of the $r-(s-1+c)$ unused preambles. This occurs with probability $(r-(s-1+c)) / r$.

2) Being the system in $(s+1, c-1)_{n-1}$, the $n$th UE chooses one of the $s+1$ preambles that were chosen by exactly one UE. This occurs with probability $(s+1) / r$.

3 ) Being the system in $(s, c)_{n-1}$, the $n$th UE chooses one of the $c$ preambles that were already chosen by more than one UE. This occurs with probability $c / r$.

\section{Estimation of the Number of Contending UEs}

The access class barring (ACB) and the extended access barring (EAB) schemes have been devised by the 3GPP to control the congestion in LTE-A cellular networks when a massive number of UEs try to access the network simultaneously [20]. To adapt the configuration of these congestion control mechanisms to the traffic load in order to achieve their optimal performance, the number of UEs attempting to access the eNB at each RAO is required [21].

In the following, we design a series of estimators of the number $N$ of contending UEs in a RAO. For this, we use two different approaches: maximum likelihood (ML) and Bayesian. Additionally, we contemplate two cases according to the information available at the eNB. In the first one, the eNB can only observe the number of successful preamble transmissions $s$. In the second one, the eNB can observe both the number of successful $s$ and the number of collided preambles $c$.

To facilitate notation, we use $\hat{N}$ to refer to a general estimator of $N$ and $x$, or $X$ if it is regarded as random variable, to the observed information by the eNB.

Then, the ML estimator of $N$ provided that a sample $x$ is observed, is simply given as

$$
\operatorname{ML}(x) \triangleq \underset{n}{\arg \max } P_{n}(x)
$$

For a given loss function $L(N, \hat{N})$, the Bayes estimator is defined as the decision rule that minimizes the posterior expected loss:

$$
\begin{aligned}
\hat{N}_{L}(x) \triangleq \underset{m}{\arg \min } \mathbb{E} & {[L(N, m) \mid X=x] } \\
& =\underset{m}{\arg \min } \sum_{n} L(n, m) \operatorname{Pr}(n \mid x) .
\end{aligned}
$$


In this paper, we select the relative estimation error as the loss function, that is,

$$
L(N, \hat{N}) \triangleq \frac{|N-\hat{N}|}{N} .
$$

Then, the resulting Bayesian estimator is given as

$$
\mathrm{B}(x) \triangleq \frac{1}{2}\left(\max \left\{m: S_{x}(m) \leq 0\right\}+\min \left\{m: S_{x}(m) \geq 0\right\}\right),
$$

where

$$
S_{x}(m) \triangleq \sum_{n=1}^{m} \frac{1}{n} P_{n}(x) \pi(n)-\sum_{n>m} \frac{1}{n} P_{n}(x) \pi(n)
$$

and $\pi(n) \triangleq \operatorname{Pr}(N=n)$ is the prior distribution of $N$. Please observe that in our approach we assume that no information is available on the value of $N$. Consequently, based on the principle of indifference [22], [23], we will consider $\pi(n)$ to be a uniform distribution over $\left\{1,2, \ldots, N_{\max }\right\}$, which is a typical choice as a non-informative prior in a Bayesian framework [23]; and $N_{\max }$ is the maximum number of contending UEs in a RAO. This value must be selected according to the maximum expected load. As it will be described in Section IV-B, the maximum number of contending UEs in highly congested scenarios and with no congestion control in place has been observed to be in the order of a few hundreds [8], [9]. Note that the use of a non-informative prior has minimal influence on the final estimation [23]. Equation (13) then becomes

$$
S_{x}(m)=\sum_{n=1}^{m} \frac{1}{n} P_{n}(x)-\sum_{n=m+1}^{N_{\max }} \frac{1}{n} P_{n}(x) .
$$

As noted above, two different cases are contemplated according to the information available at the eNB. In the first case, the observed sample $x$ is the number of successful preamble transmissions $s$; and in the second case, it is the number of both successful and collided preambles $(s, c)$. To differentiate these two cases, the estimator will be denoted as $\hat{N}_{1}$ and $\hat{N}_{2}$, respectively.

In the numerical results presented in Section IV, it can be observed that while the estimators introduced so far provide an acceptable accuracy, they are biased. In what follows, we propose a modification of $\hat{N}$ with the aim of reducing the bias to obtain a more accurate estimator.

Let

$$
\mu(n) \triangleq \mathbb{E}[\hat{N} \mid N=n]=\sum_{x} \hat{N}(x) P_{n}(x) .
$$

If $\hat{N}$ were not biased, $\mu(n)=n$, but in our case, $\mu(n)<n$ with $\hat{N}_{1}$ and $\mu(n)>n$ with $\hat{N}_{2}$ for most values of $n$.

Let us assume for the moment that the value of $N$ is known: $N=n$. Then, the estimator

$$
\widetilde{N} \triangleq \frac{\hat{N}}{\mu(n) / n}
$$

would be clearly unbiased. However, $\widetilde{N}$ is not a realizable estimator, because it depends on the actual value of $N$, which the estimator is supposed to estimate.

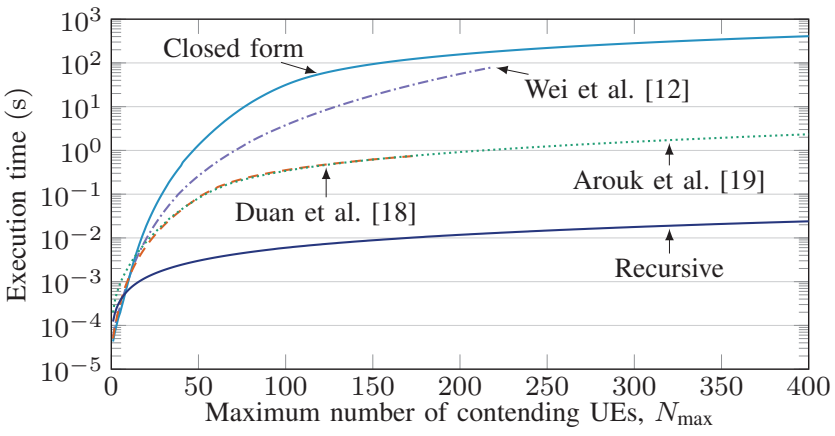

(a)

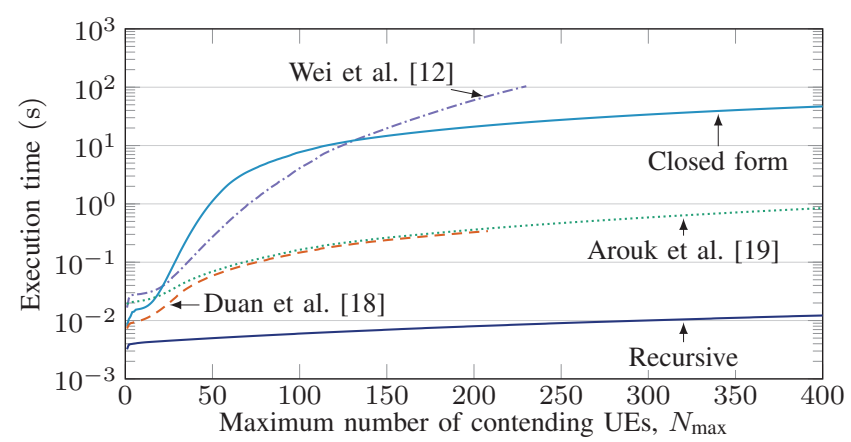

(b)

Figure 1. Execution time in seconds when the number of preambles is (a) $r=54$ and (b) $r=30$ as a function of the maximum number of contending UEs, $N_{\max }$.

Since the true value of $N$ is actually unknown, we can make the approximation $N=n \approx \hat{N}$. In this way we obtain the refined estimator

$$
\hat{N}^{*} \triangleq \frac{\hat{N}}{\mu(\hat{N}) / \hat{N}}=\frac{\hat{N}^{2}}{\mu(\hat{N})} .
$$

As demonstrated in the following section, applying the refinements described above to both $\hat{N}_{1}$ and $\hat{N}_{2}$ increases their accuracy for most values of $N$. The new estimators are denoted as $\hat{N}_{1}^{*}$ and $\hat{N}_{2}^{*}$.

\section{Numerical Results}

In this section, two different types of numerical experiments to evaluate the efficacy of our contributions are conducted. First, we compare the computational cost of our methods with that of some related methods that have recently appeared in the literature [12], [18], [19]. However, it is important to emphasize that our methods and those used as a basis for comparison do not provide the same information: ours calculate the joint PDF of successes and collisions, whereas the other methods only calculate the PDF of successes. Second, we evaluate the accuracy of the designed estimators. The results of these experiments are presented in Section IV-A and Section IV-B, respectively.

\section{A. Computational Cost Comparison}

In addition to our methods, we implemented the methods proposed in [12], [18], [19] and analyzed the computational cost of all of them in terms of execution time. Our 
implementations were done in MATLAB 2015b, and were run on a PC with MS Windows 8.1 (64 bit), an Intel Core i7-4702MQ processor, $2.2 \mathrm{GHz}$ and $16 \mathrm{~GB}$ RAM. During the execution of our MATLAB code, no other processes with a relevant CPU usage were run. We have also studied the cost in terms of the number of floating point operations. Here only the execution time results are shown since both metrics lead to the same conclusions.

Fig. 1 shows the execution time that each method requires to obtain the PDFs that are needed in the estimation when the maximum number of contending UEs in a RAO is $N_{\max }$. The curves corresponding to two of the studied methods were interrupted at the point in which these methods stop generating valid results due to numerical stability issues.

Fig. 1a illustrates the computational cost when the number of available preambles for the contention-based random access procedure is $r=54$. This is the most typical scenario according to the LTE-A specification [5], [24]. In addition, Fig. 1b illustrates the computational cost when $r=30$. The reason for selecting this value is that the random access procedure defined in the NB-IoT standard is similar to that in LTE-A, but only $r=48$ preambles are available [25]. Concretely, NB-IoT UEs with an acceptable wireless connection to the eNB belong to coverageenhancement (CE) level zero and perform only one preamble repetition per access attempt. The remaining UEs belong to $\mathrm{CE}$ levels one and two, and perform several preamble repetitions to decrease the probability of an access failure due to a wireless channel error. Thus, setting $r=30$ for UEs in CE level zero, which is expected to contain most of the UEs, seems adequate as it allows for the reservation of the remaining 18 preambles for UEs in higher CE levels.

The results in Fig. 1 show that, in addition to providing more detailed information, $P_{n}(s, c)$ vs. $P_{n}(s)$, our formulations also offer computational advantages in terms of both numerical stability and computational cost. In Fig. 1a, it can be observed that our recursive method is up to 6000,70 , and 100 times faster than that of the formulae presented in [12], [18], [19], respectively. Likewise, in Fig. 1b, it can be observed that our recursive method is $1.2 \times 10^{4}$, 40 , and 70 times faster.

\section{B. Accuracy of the Estimators}

In this section, the results of assessing the accuracy of the proposed estimators are presented. Different values for the number of available preambles $r$ were used, and it was observed that the results were qualitatively similar for all of them. Therefore, next we show and analyze the results corresponding to $r=54$.

Fig. 2 shows the estimated value of $N$ with the ML and Bayesian estimators. As can be seen, the accuracy of the estimation is much higher when $(s, c)$ are known (i.e., $\hat{N}_{2}$ ) than when only $s$ is known (i.e., $\hat{N}_{1}$ ). Fig. 2 also shows that the estimators are biased, which led to the formulation of the refined estimators $\hat{N}_{1}^{*}$ and $\hat{N}_{2}^{*}$ in (17).

In real implementations, the number of UEs within a single cell could be significantly large. Therefore, to assess the accuracy of our estimators, we varied the number of UEs that transmit a preamble in a RAO from one UE up to 300 . This



Figure 2. Expected value of the ML and Bayesian estimators given the number of contending UEs.



(a)

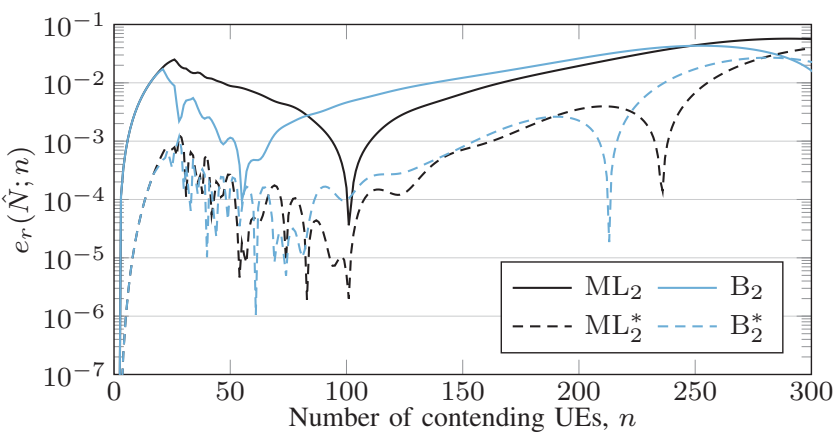

(b)

Figure 3. Expected relative error when $r=54$ using: (a) $P_{n}(s)$ and (b) $P_{n}(s, c)$.

latter value was selected as it is approximately the maximum number of contending UEs per RAO in the most congested scenario described by the 3GPP [8], [24].

Fig. 3 illustrates the expected relative estimation error as a function of $n$, which is defined as

$$
e_{r}(\hat{N} ; n) \triangleq \mathbb{E}[L(N, \hat{N}) \mid N=n]=\sum_{x} \frac{|n-\hat{N}(x)|}{n} P_{n}(x),
$$

for all the proposed estimators.

Fig. 3a shows the expected relative error of $\hat{N}_{1}$ and $\hat{N}_{1}^{*}$, that is, only $s$ is known to the eNB. We observe that the ML estimator is accurate for $N \leq r$, except for $N=2$, where there is a sharp peak. This same peak is observed with the Bayesian estimator at exactly $N=2$. It is worth noting that the accuracy of the Bayesian estimator is lower than that of the ML estimator when $N \leq r$, but the opposite occurs for $r<N<100$. For $N \geq 100$ both estimators present a similar accuracy. However, it should be borne in mind that to properly 
observe the power of the Bayesian estimation one would have to consider a sequential method that enhances the accuracy of the estimation by incorporating observations as they are made in subsequent RAOs. Devising and studying such sequential estimation method is beyond the scope of this paper and is left for future research. Furthermore, the refinements to reduce the bias of the estimators sharply increase their accuracy for most values of $N \leq 100$, where the expected error of the initial estimator is usually lower than 20 percent. Conversely, when the expected error in the initial approximation surpasses 50 percent, the refined estimation presents a similar accuracy.

Fig. 3b shows the expected relative error of $\hat{N}_{2}$ and $\hat{N}_{2}^{*}$. We observe that the initial estimation results using $\hat{N}_{2}$ are highly accurate: $e_{r}\left(\hat{N}_{2} ; n\right) \approx 5.8$ percent in the worst case. After refining these results using $\hat{N}_{2}^{*}$, we obtain even more accurate estimations: $e_{r}\left(\hat{N}_{2}^{*} ; n\right) \leq 4$ percent for all the values of $n$.

It is worth noting that our proposed estimators provide even more accurate results under light-load conditions. Concretely, when $N \leq 100$. This has great significance as the purpose of congestion control mechanisms, such as the ACB and EAB schemes, is to maintain a light signaling traffic load. For instance, the RACH capacity is achieved when $\mathbb{E}[N]=$ $[\log (r /(r-1))]^{-1}<r$ [26]. Hence, this is the optimal point of operation.

\section{Conclusions}

We presented expressions to obtain the joint probability distribution (PDF) for the number of successful and collided preambles in a random access opportunity (RAO) of an LTE-A network. Based on the available information at the eNB regarding the access attempts, we designed a series of maximum likelihood and Bayesian estimators of the number $N$ of contending MTC devices in a RAO.

Numerical results showed that our formulations are computationally efficient and can be used to accurately estimate the number of contending MTC devices even for heavy network loads. The proposed approaches to determine the joint PDF and to estimate $N$ can be exploited to design novel congestion control schemes, and to formulate optimization problems to set the parameters of these schemes appropriately.

In practice, a significant correlation between the number of contending UEs in consecutive RAOs is to be expected, which could be used to enhance the estimation. As a future work, we intend to extend the Bayesian estimation approach and to devise a sequential method that enhances the accuracy of the estimation by incorporating observations as they are made in subsequent RAOs.

\section{REFERENCES}

[1] Architecture Enhancements to Facilitate Communications with Packe Data Networks and Applications, 3GPP, TS 23.682, Sep. 2017.

[2] Ericsson. (2017, Nov.) Ericsson mobility report. [Online]. Available: https://www.ericsson.com/en/mobility-report/reports/november-2017

[3] D. T. Wiriaatmadja and K. W. Choi, "Hybrid random access and data transmission protocol for machine-to-machine communications in cellular networks," IEEE Trans. Wireless Commun., vol. 14, no. 1, pp. 33-46, Jan. 2015
[4] M. Condoluci, M. Dohler, G. Araniti, A. Molinaro, and K. Zheng, "Toward 5G densenets: architectural advances for effective machinetype communications over femtocells," IEEE Commun. Mag., vol. 53, no. 1, pp. 134-141, Jan. 2015.

[5] Medium Access Control (MAC) Protocol Specification, 3GPP, TS 36.321, Sep. 2017.

[6] Physical Channels and Modulation, 3GPP, TS 36.211, Sep. 2017.

[7] D. C. Chu, "Polyphase codes with good periodic correlation properties," IEEE Trans. Inform. Theory, vol. 18, Jul. 1972.

[8] L. Tello-Oquendo, I. Leyva-Mayorga, V. Pla, J. Martinez-Bauset, J. R. Vidal, V. Casares-Giner, and L. Guijarro, "Performance Analysis and Optimal Access Class Barring Parameter Configuration in LTE-A Networks With Massive M2M Traffic," IEEE Trans. Veh. Technol., vol. 67, no. 4, pp. 3505-3520, Apr. 2018.

[9] I. Leyva-Mayorga, L. Tello-Oquendo, V. Pla, J. Martinez-Bauset, and V. Casares-Giner, "On the accurate performance evaluation of the LTEA random access procedure and the access class barring scheme," IEEE Trans. Wireless Commun., vol. 16, no. 12, pp. 7785-7799, Dec. 2017.

[10] Radio Resource Control (RRC), Protocol specification, 3GPP, TS 36.331, Sep. 2017.

[11] Feasibility Study for Further Advancements for E-UTRA, 3GPP, TR 36.912, Mar. 2017.

[12] C. Wei, G. Bianchi, and R. Cheng, "Modeling and analysis of random access channels with bursty arrivals in OFDMA wireless networks," IEEE Trans. Wireless Commun., vol. 14, no. 4, pp. 1940-1953, Apr. 2015.

[13] D. P. Bertsekas, R. G. Gallager, and P. Humblet, Data networks. Prentice-Hall International New Jersey, 1992, vol. 2.

[14] O. Arouk and A. Ksentini, "Multi-channel slotted ALOHA optimization for machine-type-communication," in Proc. 17th ACM Int. Conf. Modeling, Anal. and Simulation of Wireless and Mobile Syst. (MSWiM), Sep. 2014, pp. 119-125.

[15] A. Laya, L. Alonso, and J. Alonso-Zarate, "Is the random access channel of LTE and LTE-A suitable for M2M communications? a survey of alternatives," IEEE Commun. Surveys Tuts., vol. 16, no. 1, pp. 4-16, First Quarter 2014.

[16] J. Choi, "On the adaptive determination of the number of preambles in RACH for MTC," IEEE Commun. Lett., vol. 20, no. 7, pp. 1385-1388, Jul. 2016.

[17] C.-H. Wei, R.-G. Cheng, and S.-L. Tsao, "Modeling and estimation of one-shot random access for finite-user multichannel slotted ALOHA systems," IEEE Commun. Lett., vol. 16, no. 8, pp. 1196-1199, Aug. 2012.

[18] S. Duan, V. Shah-Mansouri, Z. Wang, and V. W. S. Wong, "D-ACB: Adaptive congestion control algorithm for bursty M2M traffic in LTE networks," IEEE Trans. Veh. Technol., vol. 65, no. 12, pp. 9847-9861, Dec. 2016.

[19] O. Arouk, A. Ksentini, and T. Taleb, "How accurate is the RACH procedure model in LTE and LTE-A?" in Proc. Int. Wireless Commun. and Mobile Computing Conf. (IWCMC), Sep. 2016, pp. 61-66.

[20] Service Accessibility, 3GPP, TS 22.011, Sep. 2017.

[21] R. G. Cheng, J. Chen, D. W. Chen, and C. H. Wei, "Modeling and analysis of an extended access barring algorithm for machine-type communications in LTE-A networks," IEEE Trans. Wireless Commun., vol. 14, no. 6, pp. 2956-2968, Jun. 2015.

[22] J. O. Berger, The robust Bayesian viewpoint. Purdue University. Department of Statistics, 1982.

[23] A. R. Syversveen, "Noninformative Bayesian priors. Interpretation and problems with construction and applications," Preprint Statistics, vol. 3, 1998. [Online]. Available: https://www.math.ntnu.no/preprint/statistics/1998/S3-1998.ps

[24] Study on RAN Improvements for Machine Type Communications, 3GPP, TR 37.868, Sep. 2011.

[25] Physical Layer Procedures, 3GPP TS 36.213, Apr. 2017.

[26] T. Lin, C. Lee, J. Cheng, and W. Chen, "PRADA: prioritized random access with dynamic access barring for MTC in 3GPP LTE-A networks," IEEE Trans. Veh. Technol., vol. 63, no. 5, pp. 2467-2472, Jun. 2014. 




Luis Tello-Oquendo (S'08) received the electronic and computer engineering degree (Hons.) from Escuela Superior Politécnica de Chimborazo (ESPOCH), Ecuador, in 2010, the M.Sc. degree in telecommunication technologies, systems, and networks, and the Ph.D. degree (Cum Laude) in telecommunications from Universitat Politècnica de València (UPV), Spain, in 2013 and 2018, respectively. In 2011, he was a Lecturer with the Facultad de Ingeniería Electrónica, ESPOCH. From 2013 to 2018 he was Graduate Research Assistant with the Broadband Internetworking Research Group, UPV. From 2016 to 2017 he was a Research Scholar with the Broadband Wireless Networking Laboratory, Georgia Institute of Technology, Atlanta, GA, USA. He is currently a Research Faculty with the College of Engineering, Universidad Nacional de Chimborazo. His research interest include machine type communications, wireless software-defined networks, 5G and beyond cellular systems, Internet of Things, machine learning. He is a member of the IEEE and ACM. He received the Best Academic Record Award from the Escuela Técnica Superior de Ingenieros de Telecomunicación, UPV, in 2013, and the IEEE ComSoc Award for attending the IEEE ComSoc Summer School at The University of New Mexico, Albuquerque, NM, USA, in 2017.

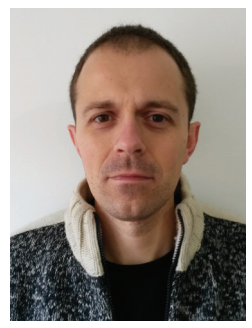

Vicent Pla received the Telecommunication Engineering (B.E. \& M.E.) and Ph.D. degrees from the Universitat Politècnica de València (UPV), Spain, in 1997 and 2005, respectively, and the B.Sc. in Mathematics from the Universidad Nacional de Educación a Distancia (UNED), Spain, in 2015. In 1999, he joined the Department of Communications at the UPV, where he is currently a Professor. His research interests lie primarily in the area of modeling and performance analysis of communication networks During the past few years, most of his research activity has focused on traffic and resource management in wireless networks. In these areas he has published numerous papers in refereed journals and conference proceedings, and has been an active participant in several research projects.

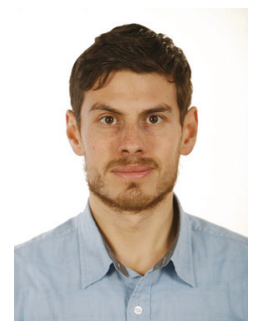

Israel Leyva-Mayorga received the B.Sc. degree in telematics engineering and the M.Sc. degree (Hons.) in mobile computing systems from the Instituto Politécnico Nacional (IPN) in Mexico City, Mexico, in 2012 and 2014, respectively. Since 2015, he has been working toward the Ph.D. degree in telecommunications at the Department of Communications, Universitat Politècnica de València, Valencia, Spain, where he was a visiting researcher in 2014. He was also a visiting researcher at the Deutsche Telekom Chair of Communication Networks, Technische Universität Dresden, Dresden, Germany, from late 2017 to mid 2018. His research interests include cellular networks, wireless sensor networks, random access protocols, mMTC, URLLC, and network coding schemes.

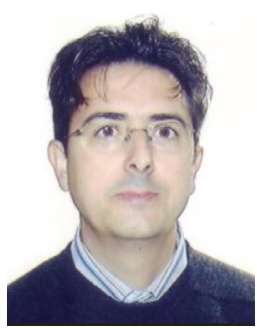

Jorge Martinez-Bauset received the Ph.D. degree from the Universitat Politècnica de València (UPV), Valencia, Spain, in 1997. He also received the 1997 Alcatel Spain Best Ph.D. Thesis Award in Access Networks. He is currently a Professor with the UPV. From 1987-1991, he was with QPSX Communications, Perth, Australia, working with the team that designed the first IEEE 802.6 MAN. Since 1991, he has been with the Department of Communications, UPV. His research interests are in the area of performance evaluation and traffic control

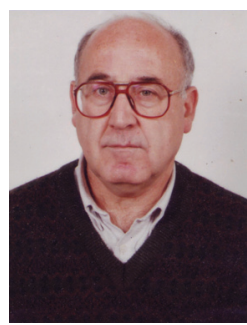

Vicente Casares-Giner (M'75-LM'17) assistant professor (1974), associate professor (1985), and full professor (1991). He obtained the Telecommunication Engineering degree in October 1974 from Escuela Técnica Superior de Ingenieros de Telecomunicación-Universidad Politécnica de Madrid (ETSITUPM) and the Ph.D. in Telecommunication Engineering in September 1980 from ETSIT-Universitat Politècnica de Catalunya (ETSITUPC), Barcelona. During the period 1974-1983 he worked on problems related to signal processing, image restoration, and propagation aspects of radio-link systems. In the first half of 1984 he was a visiting scholar at the KTH Royal Institute of Technology in Stockholm, dealing with digital switching and concurrent programming for Stored Program Control (SPC) telephone systems. From September 1, 1994 until August 31, 1995, he was a visiting scholar at WINLAB-Rutgers UniversityUSA, working with random access protocols in wireless networks, wireless resource management, and land mobile trunking system. During the 90's he worked in traffic and mobility models in several European Union (EU) projects. Since September 1996, he is at ETSIT-Universitat Politècnica de València (ETSIT-UPV), Valencia, Spain. During the 00's and 10's he has been involved in several National and EU projects. Professor V. Casares-Giner has authored several papers in international magazines and conferences, such as, IEEE, Electronic Letters, Signal Processing, EURASIP-EUSIPCO, International Teletraffic Conference (ITC), Wireless conferences, IEEE (ICASSP, ICC, ICUPC, ICNC,...). He has served as General co-chair in the ISCC 2005, in the NGI-2006 and as TPC member in several conferences and workshops (Networking 2011, GLOBECOM 2013, ICC 2015, VTC 2016,...). His main interest is in the area of performance evaluation of wireless systems, in particular random access protocols, system capacity and dimensioning, mobility management, cognitive radio and wireless sensor networks.

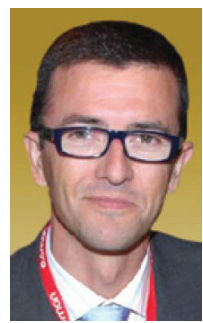

Luis Guijarro received the M.Eng. and Ph.D. degrees in Telecommunications from the Universitat Politècnica de València (UPV), Spain. He is an Associate Professor in Telecommunications Policy with the UPV. He published the book "The Electronic Communications Policy of the European Union." $\mathrm{He}$ researched in traffic management in ATM networks and in e-Government, and his current research is focused on economic modeling of telecommunication service provision. $\mathrm{He}$ has contributed in the areas of peer-to-peer interconnection, cognitive radio networks, search engine neutrality, wireless sensor networks, and $5 \mathrm{G}$. 